\title{
ASPECTOS NUTRICIONALES DE PECES ORNAMENTALES DE AGUA DULCE
}

\author{
Juan Sebastian Velasco-Garzón ${ }^{1}$, Mariana Catalina Gutiérrez-Espinosa ${ }^{2}$
}

\author{
1Zootecnista, cMSc Acuicultura. E-mail: juan.velasco@unillanos.edu.co \\ 2Zootecnista, cDra en Ciencias Agrarias. E-mail. mariana.gutierrez@unillanos.edu.co
}

${ }^{1,2}$ Facultad de Ciencias Agropecuarias y Recursos Naturales. Universidad de los Llanos. Grupo de Investigación en Acuicultura y Limnología. Villavicencio, Meta, Colombia.

\section{RESUMEN}

La comercialización de peces ornamentales se ha convertido en una actividad económica de gran importancia para la acuicultura a nivel mundial. Este documento busca recopilar y destacar la importancia de los aspectos nutricionales como son tipo de alimento, nutrientes esenciales y los diferentes componentes que favorecen el crecimiento. Es escaso el conocimiento sobre el requerimiento de nutrientes en peces ornamentales, situación que ha llevado a extrapolar la información disponible de peces de consumo, dejando de lado los requisitos específicos de las diferentes especies, afectando negativamente el rendimiento del crecimiento, el fenotipo y la fisiología. Los requerimientos proteicos que se encuentran reportados para algunas de las especies entre 25 y $53 \%$ PC. Los pigmentos, aunque no son nutrientes son considerados de gran importancia en la formulación de dietas para peces ornamentales, debido a que en algunas especies resaltan e intensifican los colores generando un incremento en su valor de venta.

Palabras clave: Peces tropicales, requerimientos nutricionales, alimento, minerales, proteína y energía.

Recibido: 21 de febrero de 2019. Aceptado: 15 de Julio de 2019.

Received: February 21, 2019. Accepted: July 15, 2019.

\section{NUTRITIONAL ASPECTS OF FRESHWATER ORNAMENTAL FISH}

\begin{abstract}
The commercialization of ornamental fish has become an economic activity of great importance for aquaculture worldwide. This document seeks to collect and highlight the importance of nutritional aspects such as the type of food, the essential nutrients and the different components that promote growth. There is little knowledge about the requirement of nutrients in ornamental fish, situation that has led to extrapolate the available information of farmed fish, leaving aside the specific requirements of the different species, negatively affecting the performance of growth, phenotype and physiology. The protein requirements that are reported for some of the species between 25 and 53\% PC. The pigments, although they are not nutrients, are considered of great importance in the formulation of diets for ornamental fish, because in some species they highlight and intensify the colors, generating an increase in their sale value.
\end{abstract}

Keywords: Tropicals fish, nutritional requirements, food, minerals, protein, energy.

Cómo citar este artículo: J. Velasco, M. Gutiérrez. "Aspectos nutricionales de peces ornamentales de agua dulce”, Revista Politécnica, vol. 15, no.30, pp82-93, 2019. DOI: 10.33571/rpolitec.v15n30a8 


\section{INTRODUCCIÓN}

En los últimos años la comercialización de peces ornamentales se ha convertido en una actividad de gran importancia para la acuicultura a nivel mundial, en donde se reconoce a países como Singapur, España, Japón, Malasia e Indonesia como los mayores productores y exportadores de peces de agua dulce [1], [2]. Para el 2013, Colombia se ubicó en la posición número 12 en el Rankin mundial de los países exportadores con 7,6 USD millones y más de 444 especies de agua dulce aprovechables comercialmente [3], [4]. Donde más del $70 \%$ de peces ornamentales exportados proceden principalmente de los ríos Orinoco, Meta, Guaviare, Vichada e Inírida, reportando que el 90\% son capturados del medio natural. Lo que ha generado una disminución considerable de las poblaciones ícticas con potencial económico, un panorama atribuido principalmente al desconocimiento de la biología básica de las especies y la falta de estudios que permitan el desarrollo de sistemas productivos a gran escala [1], [5]. La creciente demanda y la fuerte presión sobre los recursos naturales han llevado al establecimiento de granjas especializadas en aspectos nutricionales, reproductivos y cría en cautiverio de peces ornamentales con un interés creciente en las especies tropicales (Poecilia reticulata, Xiphophorus helleri, Pterophyllum scalare, entre otras).

Para el caso de Singapur la producción de peces ornamentales es considerada una industria de gran escala e importancia [6]. Sin embargo, en Colombia la producción es manejada en pequeñas granjas basadas en conocimientos empíricos que no siempre generan resultados económicos favorables, atribuido a que es una actividad productiva fundamentada por un pobre desarrollo investigativo [5].

A diferencia del aumento en la popularidad, la escala de afición y el incremento de especies con potencial que tiene la industria de los peces ornamentales, los aspectos relacionados a la biología básica de las especies como la nutrición y alimentación han recibido muy poca atención, y aún se desconocen las exigencias nutricionales en muchas especies. Lo que conlleva a que alimentos formulados para peces de consumo como la tilapia y truchas sean usados en la alimentación de peces ornamentales, causando una extrapolación de requerimientos nutricionales entre especies [7], [8], [9], [10], [11].

Este documento busca recopilar información que destaque la importancia de los diferentes aspectos nutricionales como el tipo de alimento, los nutrientes esenciales y los diferentes componentes que favorecen el crecimiento.

\section{TIPOS DE ALIMENTO}

Los peces, en contraste con la mayoría de los otros vertebrados, consumen una gran variedad de alimentos y muestran diferentes hábitos alimenticios. Según la naturaleza del alimento ingerido, se pueden diferenciar tres categorías cuyos límites son imprecisos: a.) herbívoros (incluyendo detritívoros), b.) Carnívoros y c.) Omnívoros [12], [13].

En condiciones naturales los peces escogen los alimentos según sus características como la estructura, su movilidad, tamaño de partícula, color, sabor, olor y disponibilidad, sin embargo, pueden generar algún tipo de preferencias de acuerdo con la especie, la época del año y las condiciones ambientales [14], [15]. La alimentación y nutrición son esenciales para la salud y supervivencia de los peces ornamentales. Diferentes autores han demostrado que la cantidad requerida de alimento diario para mantener el peso corporal varía según la especie, reportando el consumo de $2.4 \%$ del peso corporal/día para Danio rerio, $1.9 \%$ para Paracheidon innesi, menor de $1.5 \%$ para Trichopodus microlepsis y $1 \%$ para Pelvicachromis pulcher [16].

En juveniles, alevinos y reproductores el alimento balanceado es considerado una excelente alternativa, a pesar de que en Colombia no existen muchos estudios que describan las exigencias nutricionales de la gran variedad de especies con potencial productivo. Sin embargo, en la práctica se obtienen buenos resultados suministrando las dietas balanceadas para tilapias de $38 \%$ a $45 \%$ de proteína cruda, dependiendo el tamaño de los peces estas dietas deben ser molidas para romper el pellet [5], [8]. Asimismo, la suplementación con alimento vivo mejora los resultados productivos de especies como Paracheirodon axelrodi, Hyphessobrycon sweglesi, Nematobrycon palmeri, Apistogramma macmasteri, Apistogramma 
cacatuoides, Apistogramma iniridae, Mykrogeophagus ramirezi, entre otras [5], [17].

\section{Alimento natural}

Los peces en el ambiente natural logran balancear su dieta seleccionando entre los diversos alimentos disponibles los que mejor satisfacen sus necesidades nutricionales y rara vez se observan señales de deficiencias de nutrientes [18]. La alimentación de larvas constituye uno de los principales problemas en la acuicultura, debido a que, en esta etapa de desarrollo es necesario que las dietas sean pequeñas partículas constantes, abundantes, con alto valor nutricional, bien balanceadas y en la mayoría de las especies que el alimento tenga movimiento [8], [19]. Por lo tanto, en la fase de larvicultura y alevinaje la alimentación está basada principalmente en algas $(2-30 \mu \mathrm{m})$, rotíferos $(100-300 \mu \mathrm{m})$, protozoos $(40-250 \mu \mathrm{m})$, crustáceos (200-12000 $\mu \mathrm{m})$, nematodos (500$2500 \mu \mathrm{m})$, oligoquetos $(10-85 \mathrm{~mm})$ y "peces forrajeros" como los guppys [5], [8]. Esta dieta viva estimula el comportamiento cazador de los peces (actividad predadora), es fuente de enzimas que contribuyen a la digestión de los peces y existe gran diferencia entre la asimilación del alimento vivo $y$ el concentrado comercial causado principalmente por la digestibilidad de la proteína [8], [17], [20], [21].

Actualmente en la acuicultura se cuenta con una gran variedad de organismos considerados como alimento vivo, debido a su valor nutritivo, alta disponibilidad, fácil reproducción, gran abundancia, diferentes tamaños, cuerpo blando, ciclo de vida corto y movilidad, entre ellos se encuentran especies como: Artemia franciscana, Daphnia pulex, Eisenia foetida, Spirulina sp., Moina macrocopa, Brachionus plicatilis y Tubifex tubifex, [18], [19], [20]. Con este sistema de alimentación el crecimiento de los peces dependerá principalmente del consumo, la disponibilidad, la calidad y el enriquecimiento que tenga el alimento ofrecido [22].

El alimento vivo proporciona tanto micro como macronutrientes, específicamente presentan un adecuado balance de aminoácidos y ácidos grasos esenciales los cuales requieren las larvas de los peces, permitiendo así que están tengan un adecuado desarrollo cuando lo consumen y entre otras ventajas que tiene sobre el alimento inerte o artificial es que mantiene sus características nutritivas durante tiempos prolongados y se distribuye por toda la superficie del contenedor gracias a que se dispersa haciendo que todos los individuos puedan consumirlo [21].

En la Tabla 1 se presenta el análisis proximal y los valores energéticos de organismos más utilizados como alimento vivo para el cultivo y reproducción de peces ornamentales.

Tabla 1. Análisis proximal y valores energéticos de organismos utilizados como alimento vivo [8], [17], [18], [22]

\begin{tabular}{lcccc}
\hline \multirow{2}{*}{$\begin{array}{l}\text { Alimento } \\
\text { natural }\end{array}$} & \multirow{2}{*}{$\begin{array}{c}\text { MS } \\
\text { (\%) }\end{array}$} & \multicolumn{3}{c}{ MS Composición } \\
\cline { 3 - 5 } & & \multicolumn{2}{c}{$\begin{array}{c}\text { Lípidos } \\
(\%)\end{array}$} & $\begin{array}{c}\mathrm{EC} \\
(\mathrm{Kcal} / \mathrm{kg})\end{array}$ \\
\hline Fitoplancton $^{1}$ & $14-22$ & $17-31$ & $4-10$ & $2200-3800$ \\
Rotíferos $^{2}$ & 11,2 & 64,3 & $20,3-10$ & 4900 \\
Oligoquetos $^{3}$ & 7,3 & 49,3 & 19 & 5600 \\
Artemia & 11 & 61,6 & 19,5 & 5800 \\
Cladóceros $^{4}$ & 9,8 & 56,5 & 19,3 & 4800 \\
Copépodos $^{5}$ & 10,3 & 52,3 & 26,4 & 5500 \\
Insectos $^{6}$ & 23,2 & 55,9 & 18,6 & 5000 \\
Ostrácodos & 35 & 41.5 & - & - \\
Tubifex & - & $54-60$ & 14,3 & - \\
\hline MS:
\end{tabular}

MS: Materia seca, PC: Proteína cruda, EC: Energía cruda. 1.Algas: Chlorella sp, Isochrysis sp, Dunaliella sp, Chlamydomonas sp, Phaeodactylum $\mathrm{sp}$, entre otras. 2. Género Brachionus (Brachionus plicatilis, B. callyciflorus, B. rubens, B. urceolaris y B. falcatus). 3.Tubifex sp, Eudrilus sp y Enchytraeus sp. ${ }^{4}$ Los géneros Daphnia y Moina. ${ }^{5}$ Géneros Acartia, Centropages y Temora. ${ }^{6}$. Larvas de mosca (Chironomus) y zancudo (Culex, Aedes, Anopheles y Corethra).

En el cultivo de peces ornamentales, la Moina $s p$ solía ser el organismo más común en la alimentación de larvas y juveniles, sin embargo, como esta especie es cultivada en agua enriquecida con abono orgánico puede actuar como vector de parásitos, bacterias o virus [54]. Por esta razón gran parte de los productores cambiaron el uso Moina sp. por Artemia sp., considerándola como más inocua para la alimentación de los peces [24]. Sin embargo, se continúan realizando estudios con esta especie donde evalúan el efecto de diferentes tipos de alimento vivo sobre el crecimiento, sobrevivencia y brillo de Paracheirodon innesi, encontrando que los peces 
alimentados con Moina sp. presentaron menor crecimiento, sobrevivencia y brillo [23].

\section{Alimento artificial}

En los sistemas de cultivo de peces ornamentales a gran escala son utilizadas dietas completas que aportan todos los nutrientes en niveles que satisfacen las necesidades de una especie en particular [18]. El objeto de la formulación de alimentos artificiales es mezclar ingredientes en diferentes cantidades y calidades nutricionales que permita obtener dietas completas cuyos perfiles nutricionales disponibles se aproximen a las necesidades dietéticas de la especie en cuestión [25]. En la formulación es necesario reconocer factores como la especie a trabajar, la fisiología digestiva, los hábitos de alimentación, la fase de desarrollo del animal, la bio-disponibilidad de los nutrientes y la composición proximal de las materias primas, además, los alimentos deben tener propiedades físicas deseadas como el tamaño de partícula, la flotabilidad o hundimiento del alimento, la textura, el color y en algunos casos simular movimiento [18], [25], [26]. Las dietas comerciales elaboradas hoy en día son mezclas concentradas con menos del $10 \%$ de humedad que se encuentran en forma de polvo, grano o pellets dependiendo la etapa productiva del pez. Sus principales ventajas son que pueden ser almacenados por largo tiempo, permite controlar el consumo de alimento dependiendo de la etapa productiva de la especie y se pueden preparar para cumplir los requerimientos de la especies o etapa productiva específica [8], [18]. En la tabla 2 se presentan los requerimientos nutricionales generales teniendo en cuenta las diferentes etapas de desarrollo y el hábito de alimentación.

Tabla 2. Requerimientos nutricionales en las diferentes etapas de desarrollo y el hábito de alimentación en peces [8], [47].

\begin{tabular}{lcc}
\hline \multicolumn{1}{c}{ Etapa de desarrollo } & PC \% & Lípidos \% \\
\hline Alevinos & $>35$ & 8 \\
Juveniles & $>25$ & 5 \\
Reproductores & $>30$ & 5 \\
Habito de alimentación & & \\
Carnívoros & 45 & $3-6$ \\
Herbívoros & $15-30$ & $1-3$ \\
Detritívoros & $30-40$ & $2-5$
\end{tabular}

\begin{tabular}{lll} 
Omnívoros & $35-42$ & $2-5$ \\
\hline
\end{tabular}

PC: Proteína cruda

En algunas especies como por ejemplo la Arawana, Osteoglossum ferreirai se realizan protocolos de acostumbramiento del alimento vivo al concentrado balanceado. Por lo tanto, durante la primera semana las larvas son alimentadas con una dieta compuesta por $20 \%$ gupys, $70 \%$ insectos y $10 \%$ concentrado. En la segunda semana $20 \%$ gupys, $50 \%$ insectos y $10 \%$ concentrado. La tercera semana $20 \%$ insectos y $80 \%$ concentrado. Para finalizar en la cuarta semana la alimentación está basada en un $100 \%$ de alimento concentrado [5].

Aunque el alimento artificial puede llegar a reemplazar progresivamente el alimento vivo en la dieta de los peces, en la etapa de larvicultura puede conllevar a un retraso en el crecimiento [21] , es por ello que para este periodo es recomendable el suministro de alimento vivo.

\section{REQUERIMIENTOS NUTRICIONALES}

Aunque la producción y comercio de peces ornamentales son consideradas actividades muy rentables en la acuicultura, además de reconocidas a nivel mundial, hay poca información acerca de los requerimientos nutricionales de estos [26]. La gran mayoría de la bibliografía reportada se enfoca en mostrar el efecto de algunas dietas o alimentos sobre parámetros zootécnicos, pero no determinan los requerimientos [23], [28], [29], [30], [31].

El alimento contiene nutrientes y energía que son esenciales para el crecimiento, la reproducción y la salud de los peces. Las deficiencias de estas sustancias pueden reducir las tasas de crecimiento o dar lugar a enfermedades por agentes patógenos oportunistas. Por otra parte, excesos pueden causar una disminución del consumo de alimento por saciedad prolongada y poco crecimiento.

Los requerimientos dietarios en las diferentes especies se pueden establecer para la proteína, energía, aminoácidos, lípidos, carbohidratos, minerales y vitaminas [8], [26], [32]. En la tabla 3 se muestra una recopilación de diferentes ensayos en donde determinaron requerimientos de proteína, energía y lípidos en peces ornamentales de gran importancia para la acuicultura. 
Tabla 3. Requerimientos de proteína, lípidos y energía de diferentes especies de peces ornamentales.

\begin{tabular}{|c|c|c|c|c|}
\hline Especie & $\begin{array}{l}\mathrm{PC} \\
(\%)\end{array}$ & $\begin{array}{l}\text { Energía } \\
(\text { kcal } / \mathrm{kg})\end{array}$ & $\begin{array}{l}\text { Lípido } \\
\text { s (\%) }\end{array}$ & $\begin{array}{l}\text { Refer } \\
\text { encia }\end{array}$ \\
\hline $\begin{array}{l}\text { Poecilia } \\
\text { reticulata }\end{array}$ & $\begin{array}{l}30- \\
40\end{array}$ & 3129 EM & - & {$[6]$} \\
\hline Carassius & 29 & 2799 ED & - & [59] \\
\hline auratus & 53 & 4849 EC & - & [60] \\
\hline $\begin{array}{l}\text { Notemigonus } \\
\text { crysoleucas }\end{array}$ & 29 & 2716 ED & - & [59] \\
\hline Barbodes altus & 41,7 & $\begin{array}{c}4867.7 \\
\text { EC }\end{array}$ & - & [58] \\
\hline $\begin{array}{l}\text { Symphysodon } \\
\text { aequifasciata }\end{array}$ & $\begin{array}{l}44,5- \\
50,1\end{array}$ & 5171 EC & - & [64] \\
\hline $\begin{array}{l}\text { Cichlasoma } \\
\text { synspilum }\end{array}$ & 40,81 & 3700 ED & - & [61] \\
\hline $\begin{array}{l}\text { Trichogaster } \\
\text { trichopterus }\end{array}$ & 35 & $3500 \mathrm{EC}$ & 8 & [65] \\
\hline $\begin{array}{l}\text { Xiphophorus } \\
\text { helleri }\end{array}$ & 40 & $4000 \mathrm{EC}$ & 10 & [33] \\
\hline $\begin{array}{l}\text { Poecilia } \\
\text { latippina }\end{array}$ & 40 & $4000 \mathrm{EC}$ & 6 & {$[66]$} \\
\hline Arapaima gigas & 48,6 & 5645 EC & - & [57] \\
\hline Cichla sp. & $\begin{array}{l}37- \\
41\end{array}$ & 3500 ED & - & [62] \\
\hline Pterophyllum & 26 & 3100 ED & - & [63] \\
\hline scalare & $\begin{array}{l}34 \\
35\end{array}$ & $\begin{array}{l}- \\
-\end{array}$ & $\begin{array}{l}- \\
6\end{array}$ & $\begin{array}{l}{[30]} \\
{[35]}\end{array}$ \\
\hline Colisa Ialia & 25 & - & - & [34] \\
\hline $\begin{array}{l}\text { Astyanax } \\
\text { bimaculatus }\end{array}$ & $\begin{array}{l}32- \\
38\end{array}$ & 2900 ED & - & [36] \\
\hline
\end{tabular}

\section{Proteínas y aminoácidos}

Las proteínas son péptidos de elevado peso molecular, compuestas por carbono, hidrógeno, oxígeno, nitrógeno y con frecuencia azufre. Las unidades fundamentales de estas son los aminoácidos, los cuales pueden unirse entre sí por un enlace peptídico covalente entre el $\alpha$-carboxilo final de un aminoácido y el $\alpha$-amino final del otro. Los aminoácidos pueden enlazarse variando su secuencia para formar una vasta diversidad de proteínas [13] [25] [35], [36].
Las proteínas constituyen un componente básico de los tejidos de los organismos vivos, del 65 al $75 \%$ del total de materia seca del cuerpo de un pez está compuesto por esta molécula [38]. A nivel de mantenimiento, el pez requiere proteína para reponer tejidos desgastados y sintetizar productos proteicos como células epiteliales, enzimas y hormonas esenciales para el correcto funcionamiento del organismo, las cuales recirculan velozmente, es decir que están formándose y degradándose continuamente [6], [8], [13]. La capacidad del pez para sintetizar proteína de nuevo a partir de esqueletos de carbono es limitada, por lo tanto, la mayor parte de ella debe obtenerse del alimento. En el proceso fisiológico de digestión, la proteína es digerida e hidrolizada liberando aminoácidos, los cuales son absorbidos por el tracto intestinal y distribuidos por la sangre para todos los órganos y tejidos [36]. En los sistemas de producción la ingesta diaria de alimentos con niveles óptimo de proteína es fundamentales para el crecimiento y mantenimiento de los peces [8], [17], [25], [36]

Cuando se habla de requerimiento se hace referencia a la cantidad mínima necesaria que debe tener el alimento para satisfacer las necesidades de aminoácidos y lograr el máximo crecimiento en las diferentes especies [10], [25], [26]. Diversos estudios calculan los requerimientos suministrando diferentes niveles de proteína con alto valor biológico en la dieta, evaluando la respuesta en función del crecimiento de la especie en un periodo determinado de tiempo [10], [25], [37]. Por otra parte, un exceso de proteína en las dietas genera que solo una parte de esta se utilice en la formación de tejidos y el restante sea transformado en energía a partir de los aminoácidos, además incrementa la excreción de compuestos nitrogenados por heces y branquias comprometiendo la calidad del agua [33]. En el caso de los aminoácidos esenciales existen estudios que reportan el requerimiento absoluto de lisina, arginina, histidina, isoleucina, leucina, metionina, fenilalanina, treonina, triptófano, y valina demostrado en muchas especies de peces de consumo como ornamentales [17], [25], [26]. En Goldfish, C. auratus determinaron los requerimientos de lisina $(11,8 \mathrm{gr})$, metionina $(3,4 \mathrm{gr})$, leucina $(9,1 \mathrm{gr})$, treonina $(6,4 \mathrm{gr})$, arginina $(7,8 \mathrm{gr})$, isoleucina (6gr) y valina (7gr) representados en $\mathrm{gr}$ de aminoácidos por $100 \mathrm{gr}$ de proteína, obteniendo los mayores pesos en la fase de larvicultura durante un periodo corto de 20 días [6]. 


\section{Energía}

La energía no es considerada como un nutriente, sin embargo, es liberada durante la oxidación metabólica de estos. Por ello para varias especies han sido determinadas las exigencias dietarías de los nutrientes conjuntamente con las exigencias de energía. Como principio general, los animales comen para satisfacer sus necesidades energéticas esenciales (metabolismo básico, actividades rutinarias, crecimiento, reproducción) [37].

La fuente principal de energía es el sol, a través del proceso de fotosíntesis los cloroplastos de las plantas verdes capturan la energía lumínica y la fijan convirtiéndola en energía química. La energía química contenida en el alimento puede ser cuantificada en unidades de calor; la unidad básica de energía calorífica es la caloría. El total de energía contenida en una sustancia es determinado oxidándola completamente hasta bióxido de carbono. La cantidad de calor resultante de esta oxidación es conocida como calor de combustión o valor energético bruto [37].

Los peces ornamentales obtienen la mayor parte de su energía a partir de lípidos contenidos en la dieta. Sin embargo, los lípidos no deben superar el 15\% de la ingesta diaria, esto para evitar la lipidosis hepática [40]. Los peces pueden modificar su consumo de alimento dependiendo del nivel de energía de la ración ingerida, raciones con mayor nivel de energía pueden consumir cantidades menores de proteína perjudicando su desarrollo [34], [36].

La energía de cualquier alimento está influenciada por una serie de enzimas complejas que requieren de la presencia de vitaminas o minerales para su correcto funcionamiento. La energía requerida para el mantenimiento y la síntesis de proteínas en peces es menor que en mamíferos [27]. Por lo tanto, la relación proteína: energía en los peces es mayor, debido principalmente a los bajos niveles de requerimientos energéticos. El factor más importante que afecta el requerimiento de energía de un pez es la temperatura de agua. Un aumento en la temperatura del agua aumentará la temperatura corporal de los peces, lo que, acelerará las reacciones metabólicas y aumentará los requerimientos de energía de los peces [8] y [13]. En consecuencia, la cantidad de alimento requerido por un pez aumentará a medida que la temperatura del agua comience a aumentar y disminuirá a medida que la temperatura del agua comience a disminuir [34]. La Tabla 3 muestra valores de energía de dietas suministradas a algunas especies de peces ornamentales de agua dulce.

\section{Lípidos y ácidos grasos.}

Los lípidos comprenden un grupo variado de compuestos orgánicos que son insolubles en agua, pero solubles en solventes orgánicos [38]. Nutricionalmente son considerados como fuentes de energía concentrada y compuestos esenciales para el crecimiento de los peces [17] y [26]. Su digestión se da a nivel del intestino con una gran participación del hígado por la acción de enzimas digestivas (lipasas) que los divide en glicerol y ácidos grasos responsables de la liberación de energía, la actividad enzimática, procesos metabólicos, síntesis de grasa corporal, síntesis de hormonas reproductivas, flexibilidad y permeabilidad de las membranas plasmáticas [8], [28] y [35]. Fisiológicamente los ácidos grasos libres constituyen la principal fuente de combustible aerobio para el metabolismo energético del músculo de los peces [22]. La literatura sobre lípidos en peces es amplia, principalmente en peces de consumo, pero incompleta, debido a que varios aspectos de su metabolismo son desconocidos y deducidos por la homologación de conocimientos sobre los mamíferos y otros animales [37].

\section{Carbohidratos}

Son considerados biomoléculas compuestas por carbono, hidrógeno y oxígeno, cuyas funciones son de tipo estructural en las células y brindar energía inmediata. La glucosa y el glucógeno son las formas biológicas primarias de almacenamiento y consumo de energía [8].

La utilización de los carbohidratos en los peces depende principalmente del hábito de alimentación, así como también de la estructura y la función del sistema digestivo, la especie, la digestibilidad y la complejidad de almidón a suministrar [33]. Su valor nutricional varía entre las diferentes especies siendo los peces de aguas cálidas aquellos que pueden usar cantidades mayores de carbohidratos en la dieta a diferencia de los peces de agua fría y marinos [15]. 
Para el caso de los peces ornamentales no se reportan requerimientos dietarios específicos para los carbohidratos, sin embargo, si estos no son suministrados en la dieta otros compuestos como las proteínas y lípidos son catabolizados para la liberación de energía afectando directamente el crecimiento de los peces [6], [8], [26]. Por lo tanto, los carbohidratos suministrados en las dietas para peces son considerados de menor importancia como fuentes energéticas, pero pequeñas inclusiones en la dieta han demostrado que contribuyen a evitar el uso de la proteína del alimento para la liberación de energía [15]. En peces herbívoros, como el goldfish y la carpa koi utilizan la microflora presente en el intestino posterior para digerir carbohidratos complejos. La digestibilidad de carbohidratos puede variar desde un $70 \%$ en goldfish hasta un 50\% para Trichogaster microlepis (Gourami) [6], [40].

\section{Vitaminas}

Las vitaminas son compuestos orgánicos de bajo peso molecular requeridos en bajas cantidades y no son sintetizadas por los peces, por lo que es imprescindible que sean suministradas a través de los alimentos [26], [38], [42]. Actúan como cofactores enzimáticos en diferentes procesos como el metabolismo energético, la síntesis de aminoácidos, la síntesis de proteínas, la oxidación y síntesis de ácidos grasos y triglicéridos, la síntesis de hemoglobina y de las células sanguíneas, la transmisión de impulsos nerviosos, etc. y presentan acciones fisiológicas específicas esenciales para el crecimiento, reproducción y salud de los peces [26].

Las vitaminas se clasifican en liposolubles (A, D, E y K) e hidrosolubles (complejo B y C). Las liposolubles son absorbidas en el intestino al igual que los lípidos y se almacenan en el organismo cuando son ingeridas en excesos, produciendo acumulaciones en diferentes tejidos produciendo toxicidad (hipervitaminosis) [18]. Esta toxicidad está dada principalmente por a vitaminas liposolubles ya que las vitaminas hidrosolubles no son almacenadas en el organismo y la cantidad sobrante es excretada [26], [37].

La vitamina $\mathrm{C}$ es considerada como la más importante de las vitaminas, debido a su poder antioxidante y su papel en el fortalecimiento del sistema inmunológico de los peces [34], por lo que, en algunas especies se han estudiado sus efectos y sus requerimientos, por ejemplo en Astronotus ocellatus, se demostró la necesidad de una suplementación de vitamina $\mathrm{C}$ en la dieta incorporando una dosis de $25 \mathrm{mg}$ de ácido ascórbico equivalentes $/ \mathrm{kg}$ en la dieta, fue suficiente para prevenir la reducción del crecimiento y el desarrollo de los signos clínicos del escorbuto [43].

En Cichlasoma urophthalmus se observaron signos externos de deficiencia tales como la coloración obscura, opérculos cortos, hemorragias en los ojos, cabeza y aletas, erosión de la piel y aletas, pérdida de escamas, exoftalmia, abdomen inflamado, iritis, escoliosis y cambios en los huesos de la cabeza. El nivel mínimo de vitamina $C$ requerido para el crecimiento normal de esta especie fue de 40 $\mathrm{mg} / \mathrm{kg}$ de dieta y el mínimo requerido para asegurar que los peces estuvieran sanos fue de $110 \mathrm{mg} / \mathrm{kg}$ [44]. Blom et al (1990) sugieren que el requerimiento de vitamina C para Pterophylum scalare es de $360 \mathrm{mg} / \mathrm{kg}$ [45].

\section{Minerales}

Los minerales son elementos químicos inorgánicos que hacen parte estructural de los peces y son requeridos en cantidades semejantes a las exigidas por otros animales domésticos. Sin embargo, es importante resaltar que existe una gran diferencia en la forma como estos son absorbidos [46], [47] y [48]. En los animales terrestres la vía de obtención de los minerales es a través de la ingesta de alimentos y agua. En cambio, los organismos acuáticos además de obtenerlos por la ingesta, tiene la alternativa de absorberlos directamente del agua a través de las branquias y la piel [25], [26].

Los minerales se dividen de manera arbitraria en macrominerales y microminerales. Donde los macrominerales son requeridos en mayores cantidades como el calcio, fósforo, potasio, cloro, magnesio y sodio. Los microminerales son necesarios en menores cantidades como el cobre, hierro, manganeso, selenio y zinc [26], [38], [47]. Estos participan en la formación de huesos y dientes, el metabolismo energético, los componentes de los fosfolípidos en las membranas celulares, los componentes de la hemoglobina, el equilibrio osmótico, el equilibro ácido-base de la sangre, la transmisión de impulsos nerviosos, contracción muscular, osmorregulación, componentes de las hormonas, entre otros 
procesos [18], [26]. Los rangos correspondientes a los requerimientos de microminerales en peces de agua dulce son expresados en $\mathrm{mg}$ por $\mathrm{kg}$ de dieta en base seca (Tabla 4).

Tabla 4. Rangos de inclusión de minerales en peces de agua dulce [6], [47].

\begin{tabular}{lccc}
\hline \multicolumn{1}{c}{ Mineral } & Rango en Peces & $\begin{array}{c}B . \\
\text { reticulata }\end{array}$ & $\begin{array}{c}B . \\
\text { tetrazona }\end{array}$ \\
\hline Hierro & $30-170$ & 0,0008 & - \\
Cobre & $1-5$ & - & - \\
Manganeso & $2-20$ & 0,054 & - \\
Zinc & $15-40$ & 0,01 & - \\
Cobalto & $0,05-1$ & - & - \\
Selenio & $0,15-0$, & - & - \\
Yodo & $1-4$ & - & - \\
Fosforo & - & $0,53-1,03$ & 0,52 \\
\hline *Expresados en mg de minerales por kg
\end{tabular}

Establecer los requerimientos dietéticos de Calcio (Ca) en peces es difícil por la presencia de Ca en el ambiente de cría y por el hecho de que los peces puedan absorber el Ca del agua. Esta absorción del calcio a través del medio ambiente varia entre especies, el sistema endocrino, la disponibilidad en la dieta y de la concentración de $\mathrm{Ca}$ en el agua. Por lo tanto, la determinación del requerimiento de $\mathrm{Ca}$ dietético es necesario establecerlo para cada especie teniendo en cuenta el crecimiento y la mineralización ósea. Las dietas deben mantener una proporción idea de $\mathrm{Ca} / \mathrm{P}$, de lo contrario, un exceso de $\mathrm{Ca}$ o $\mathrm{P}$ puede causar problemas en el desarrollo corporal de los peces, dificultar su absorción y permanecer en el entorno de cultivo [49]. Se requiere más investigación sobre la absorción, biodisponibilidad, requerimiento y utilización no solo en cuanto al Ca sino general en minerales en peces [50] y específicamente en ornamentales.

\section{Pigmentos}

Los pigmentos son responsables por el amplio espectro de la coloración en los peces ornamentales, siendo un requisito de calidad que a su vez determina el precio de varias especies en el mercado [51], [52]. Debido a que en los peces los pigmentos no pueden ser sintetizados deben ser suministrados a través de los alimentos [26], [52].
Entre las principales clases de pigmentos se encuentran las xantofilas y los carotenoides que son utilizados en dietas prácticas para incrementar la coloración en la piel y las aletas de los peces [26], [51]. Los carotenoides son considerados como compuestos esenciales para la salud, crecimiento, metabolismo y reproducción en los peces [53], [54]. Estos se pueden encontrar presentes en numerosas variedades de plantas, algas, pequeños crustáceos y microorganismos contienen gran variedad de pigmentos de gran importancia en la industria de los peces de acuario generando intensidad en diferentes colores que van desde el amarrillo, naranja hasta los rojos intensos [53], [54]. En la tabla 5 se describen los principales pigmentos reportados en la literatura para ser usados en la fabricación de dietas para peces ornamentales y la respectiva coloración que producen.

Tabla 5. Principales pigmentantes utilizados en dietas para peces ornamentales [52].

\begin{tabular}{lc}
\hline \multicolumn{1}{c}{ Pigmentos } & Color \\
\hline Tunaxantina & Amarillo \\
Betacarotenos & Naranja \\
Alfa, Beta-Doradexanthens & Amarillo \\
Luteína & Verdoso con Amarillo \\
Zeaxantina & Amarillo con Naranja \\
Astaxantina & Rojo \\
Equinenona & Rojo \\
Taraxantina & Amarillo \\
\hline
\end{tabular}

En peces ornamentales se reporta requerimientos entre 50 a $400 \mathrm{mg} / \mathrm{L}$ de carotenos sintéticos o naturales para obtener colores intensos, similares a los obtenidos con alimento vivo [56]. Especies como Xiphophorus helleri, Pseudomungil furcatus y Cichlasoma myrnae obtuvieron una coloración más intensa usando 1,5 a $2 \%$ de carotenoides en la dieta, durante un período de alimentación de 3 semanas. En Goldfish, Carassius auratus se obtuvo la coloración más intensa agregando astaxantina de 36 a $37 \mathrm{mg} / \mathrm{Kg}$ en las dietas [52]. A su vez, implementaron alfalfa como fuente de carotenoides para obtener mayor intensidad en el color, usando el $26 \%$ de inclusión en dietas para la alimentación de goldfish, Carassius auratus [53]. En carpas de la especie Cyprinus carpio la combinación de Luteína y Betacarotenos en proporciones de 25:25 y 50:50 $\mathrm{mg} / \mathrm{kg}$ en las dietas incrementaron la coloración en esta especie, observando que al usar $50 \mathrm{mg} / \mathrm{kg}$ de 
luteína se presentaba una mayor intensidad en la coloración roja del cuerpo contribuyendo al cambio de imagen y calidad de la coloración del pez [54].

\section{CONCLUSIONES}

Es escaso el conocimiento sobre el requerimiento de nutrientes en peces ornamentales, situación que ha llevado a extrapolar la información disponible de peces de consumo, dejando de lado los requisitos específicos de las diferentes especies, afectando negativamente el rendimiento del crecimiento, el fenotipo, la fisiología y la calidad del agua.

El alimento vivo debe ser substituido paulatinamente por alimento artificial, sin embargo, en la etapa de larvicultura se recomienda no substituirlo totalmente debido a las ventajas que tiene, tales como mantener sus características nutritivas durante tiempos prolongados, se distribuye por toda la superficie del contenedor gracias a que se dispersa haciendo que todos los individuos puedan consumirlo y estimula la actividad predadora mejorar el desarrollo de los peces.

Los carbohidratos y lípidos cumplen un papel fundamental en la formulación de dietas para peces, debido a que son compuestos liberadores de energía y en proporciones adecuadas ayudan a que el pez no use la proteína como fuente de energía si no como fuente de aminoácidos para formación de tejidos y musculo generando un efecto denominado "ahorro de proteína".

Los pigmentos, aunque no son nutrientes son considerados de gran importancia en la formulación de dietas para peces ornamentales, debido a que en algunas especies resaltan e intensifican los colores generando un aumento en el valor de venta y el agrado a la hora de comercialización.

Se recomienda realizar estudios que contribuyan a la determinación de los requerimientos nutricionales de las principales especies con potencial productivo en la Orinoquia Colombiana, a su vez técnicas y tecnologías que favorezcan la producción de dichas especies en condiciones de cultivo buscando disminuir la presión de captura en los diferentes ecosistemas.

\section{AGRADECIMIENTOS}

A la Dirección General de Investigaciones de la Universidad de los Llanos por la financiación del proyecto de tesis de Maestría en Acuicultura C04F01-002-2016 "DETERMINACIÓN DE LOS REQUERIMIENTOS DE PROTEÍNA Y ENERGÍA EN JUVENILES DE MONEDA, Metinnys orinocencis (Steindachner, 1908)".

\section{REFERENCIAS BIBLIOGRÁFICAS}

[1] LEGISCOMEX. Estudio de mercado peces ornamentales en Colombia. Cartilla para el etiquetado de alimentos en EE.UU. Disponible en: https://www.legiscomex.com/BancoMedios/Docume ntos\%20PDF/estudio\%20peces\%20ornamentales\% 20completo3.pdf [consultado el 26 de febrero de 2016]

[2] Ling, K. y Lim, Y. The Status of Ornamental Fish Industry In Singapore. J. Pri Ind., 32, 59-69, 2006.

[3] Instituto Colombiano de Desarrollo Rural. Resolución 3532 de 2007. Colombia, 2007.

[4] Ortega-Lara, A., Cruz-Quintana, M. y PuentesGranada, V. Dinámica de la actividad pesquera de peces ornamentales continentales en Colombia. Serie recursos pesqueros de Colombia - AUNAP. Autoridad Nacional de Pesca y Acuicultura. Fundación FUNINDES, 2015.

[5] Landines, M., Sanabria, A. y Daza, P. Producción de peces ornamentales en Colombia. INCODER. Bogotá D.C. 2007.

[6] Sales, J. y Janssens, G. Nutrient requirements of ornamental fish. Aquatic Living Resources. 16(6), 533-541, 2003.

[7] Pannevis, C. y Earle, K. Nutrition of ornamental fish: Water soluble vitamin leaching and growth of Paracheirodon innesi. The Journal of Nutrition. 124, 2633S-2635S, 1994.

[8] Wedler, E. Introducción en la acuicultura con énfasis en los neotrópicos. 1ra edición. Santa Marta. 1998. 
[9] Earle, K.E. The nutritional requirements of ornamental fish. The Veterinary Quarterly, 17(1), S53-S55, 1995.

[10] Ribeiro, F.A., Rodrigues, L.A. y Fernandes, B.K. Desempenho de juvenis de Acará-Bandeira (Pterophyllum scalare) com diferentes níveis de proteína bruta na dieta. B. Inst. Pesca, 33(2), 195203, 2007.

[11] Stevens, C.H., Croft, D.P., Paull, G.C y Tyler, C.R. Stress and welfare in ornamental fishes: what can be learned from aquaculture. Journal of Fish Biology, 91(2), 409-428, 2017.

[12] Prejs, A. y Colomine, G. Métodos para el estudio de los alimentos y las relaciones tróficas de los peces, Universidad Central de Venezuela, Caracas; Universidad de Polonia, 1981.

[13] NRC Nutrients Requirements of Fish. National Research Council, Washington. D.C., 2011.

[14] Hahn, N.S. y Cunha, F. Feeding and trophic ecomorphology of Satanoperca pappaterra (Pisces, Cichlidae) in the Manso Reservoir, Mato Grosso State, Brazil. Brazilian Archives of Biology and Technology, 48(6), 1007-1012, 2005.

[15] Fracalossi, D. y Cyrino J. Nutriaqua: nutrição e alimentação de espécies de interesse para a aquicultura brasileira. Sociedade Brasileira de Aquiculura e Biologia Aquática, Florianópolis, 2013

[16] Pleeging, C.C.F. y Moons, C.P.H. Potential welfare issues of the Siamese fighting fish (Betta splendens) at the retailer and in the hobbyist Aquarium. Vlaams Diergeneeskundig Tijdschrift, 86, 213-223, 2017

[17] Prieto, M. y Atencio, V. Zooplancton en la larvicultura de peces neotropicales. Revista MVZ Córdoba, 13 (2), 1415-1425, 2008.

[18] Hepher, B. Nutrición de peces comerciales en estanques. Limusa S.A, México, 1993.

[19] Luna-Figueroa, J. Incorporación de larvas de mosquito Culex stigmatosoma (Diptera:Culicidae) en la dieta del pez cebra Brachidanio rerio (Pisces:Cyprinidae) y su efecto en la reproducción. Avances en Investigación Agropecuaria, 11(1), 4959, 2007.
[20] Luna-Figueroa, J. y Arce-Uribe, E. Un menú diverso y nutritivo en la dieta de peces "El Alimento Vivo". Agroproductividad, 10(9), 112-116, 2017.

[21] Luna-Figueroa, J., Arce-Uribe, E. y Figueroa, J. Ventajas e inconvenientes del uso de alimento vivo en la nutrición de peces. Inventio, 14(33), 39-43, 2018

[22] Luna-Figueroa, J., Vargas, Z. y Figueroa, J. Alimento vivo como alternativa en la dieta de larvas y juveniles de Pterophyllum scalare (Lichtenstein, 1823). Avances en Investigación Agropecuaria, 14(3), 63-72, 2010.

[23] Yunita, Y., Tang, U.M. y Pamukas, N.A. Pemberian Jenis Pakan Alami Berbeda Terhadap Pertumbuhan, Kelulushidupan Dan Kecerahan Warna Ikan Neon Tetra (Paracheirodon innesi) Oleh Jurnal Online Mahasiswa (JOM) Bidang Perikanan dan IImu Kelautan, 5, 1-10, 2018.

[24] Lim, L.C., Dhert, P., y Sorgeloos, P. Recent developments in the application of live feeds in the freshwater ornamental fish culture. Aquaculture, 227(1-4), 319-331, 2003.

[25] Tacon, A. The nutrition and feeding of farmed fish and shrimp. A training manual. 3 Feeding methods. FAO, 1988.

[26] NRC. Nutrients requirements of fish. Committee on Animal Nutrition Board on Agriculture National Research Council. National Academic Pres. Washington. D. C., 1993.

[27] Lovell, R.T. Nutrition of ornamental fish. In: Kirk's current veterinary therapy XIII: small animal practice. W.B. Saunders, Philadelphia, PA, USA., 2000.

[28] Mansour, O., Idris, M., Noor, N.M. y Das, S.K. Growth performance of tinfoil barb (Barbonymus schwanenfeldii) fry feeding with different protein content diets. AACL Bioflux 10(3), 475-479, 2017.

[29] Zuanon, J.A.S., Hisano, H., Falcon, D.R., Sampaio, F.G., Barros, M.M. y Pezzato, L.E. Digestibilidade de alimentos protéicos e energéticos para fêmeas de beta. Revista Brasileira de Zootecnia, 36(4), 987-991, 2007. 
[30] Zuanon, J.A., Salaro, A.L., Balbino, E.M., Saraiva, A., Quadros, M. y Fontanari, R. Níveis de proteína bruta em dietas para alevinos de acarábandeira. R. Bras. Zootec., 35(5), 1893-1896, 2006.

[31] Soriano, M.B. y Hernandez, D. Tasa de Crecimiento del Pez Angel Pterophyllum scalare (Perciformes: Cichlidae) en Condiciones de Laboratorio. Acta Universitaria, 12(2), 28-33, 2002.

[32] Yanong, R.P.E. Nutrition of Ornamental Fish. Veterinary Clinics of North America: Exotic Animal Practice, 2(1), 19-42, 1999.

[33] Mohanta, K. y Subramanian, S. Nutrition of Common Freshwater Ornamental Fishes. Technical Bulletin No 27. ICAR Research Complex for Goa. Old Goa-India. 2011.

[34] Yousefian, M., Gharaati, A., Hadian, M., Hashemi, S., Navazandeh, A. y Molla, A. Food Requirements and Dietary in Aquarium Fish (Review). International Journal of Plant, Animal and Environmental Sciences, 2(3), 112-120, 2012.

[35] Pai, I.K. y Altaf, M.S. Protein requirement of angel fish Pterophyllum scalare (Schultze 1823). (Perciformes: Cichlidae). Journal of Bioresources, 3(1), 30-34, 2016.

[36] Cotan, J. L. V., Lanna, E. A. T., Bomfim, M. A. D., Donzele, J. L., Ribeiro, F. B. y Serafini, M.A. Níveis de energia digestível e proteína bruta em rações para alevinos de lambari tambiú. Revista Brasileira de Zootecnia, 35(3), 634-640, 2006.

[37] Gutiérrez-Espinosa, M.C., Velasco-Garzón, J.S. y León-Morales, C.A. Revisión: necesidades nutricionales de peces de la familia Pimelodidae en Sudamérica (Teleostei: Siluriformes. Revista de Biología Tropical, 67(1), 146-163, 2019.

[38] Murray, R., Bender, D., Botham, K., Kennelly, P., Rodwell, V. y Weil, H. bioquímica ilustrada. 28 Edición. Editorial Mcgraw-Hill. 92-234, 2013.

[39] Portz, L. y Furuya, W.M. 2013. Energia, proteína e Aminoácidos. En: Nutriaqua: Nutrição e alimentação de espécies de interesse para a aquicultura brasileira (Eds Fracalossi, D; Cyrino, J.) 1a edição ampliada (pp) Aquabibo. Sociedate Brasileira de Aquacultura e Biologia Aquática. Florianapolis. 65-78, 2013.
[40] Lewbart, G.A. Clinical nutrition of ornamental fish. Seminars in Avian and Exotic Pet Medicine, 7(3), 154-158, 1998.

[41] Garcia, A.S., Goncalves, L.U., Cavalli, R.O. y Viegas, E.M.M. Lípidos. Em: Nutriaqua: Nutrição e alimentação de espécies de interesse para a aquicultura brasileira (Eds Fracalossi, D; Cyrino, J.) 1a edição ampliada (pp) Aquabibo. Sociedate Brasileira de Aquacultura e Biologia Aquática. Florianapolis. 79-100, 2013.

[42] de Borda, M. R., do Carmo, M. V., \& de Abreu, J. S. Vitaminas e minerais. En: Nutriaqua: Nutrição e alimentação de espécies de interesse para a aquicultura brasileira (Eds Fracalossi, D; Cyrino, J.) 1a edição ampliada (pp) Aquabibo. Sociedate Brasileira de Aquacultura e biologia aquática. Florianapolis. 121-166, 2013.

[43] Fracalossi, D.M., Allen, M.E., Nichols, D.K. y Oftedal, O.T. Oscars, Astronotus ocellatus, Have a Dietary Requirement for Vitamin C. The Journal of Nutrition, 128(10), 1745-1751, 1998.

[44] Chávez de Martínez, M. Vitamin C requirement of the Mexican native cichlid Cichlasoma urophthalmus (Gunther). Aquaculture, 86(4), 409416, 1990.

[45] Blom, J. H., Dabrowski, K. y Ebeling, J. Vitamin C Requirements of the Angelfish Pterophylum scalare. Journal of the World Aquaculture Society, 31(1), 115-118, 2000.

[46] Tacon, A. The nutrition and feeding of farmed fish and shrimp. - A training manual. 2. Nutrients sources and composition. FAO, Brasilia, Brasil, 1987.

[47] Watanabe T, Kiron V y Satoh S. Trace minerals in fish nutrition. Aquaculture, 151, 185-207, 1997.

[48] Zhu, L., Han, D., Zhu, X., Yang, Y., Jin, J., Liu, $\mathrm{H}$. y Xie, S. Dietary selenium requirement for ongrowing gibel carp (Carassius auratus gibelio var. CAS III). Aquaculture Research, 48(6), 2841-2851, 2016.

[49] Hossain, M.A. y Yoshimatsu, T. Dietary calcium requirement in fishes. Aquaculture Nutrition, 20(1), $1-11,2014$. 
[50] Prabhu, A.P., Schrama, J.W. y Kaushik, S.J. Mineral requirements of fish: a systematic review. Reviews in Aquaculture, 8(2), 172-219, 2014.

[51] Yi-Juan, W., Yew-Hu, C. y Chih-Hung, P. Effects of dietary supplementation of carotenoids on survival, growth, pigmentation, and antioxidant capacity of characins, Hyphessobrycon callistus. Aquaculture, 261(2), 641-648, 2006.

[52] Gupta, S., Pal, A. y Venkateshwarlu, G. Use of natural carotenoids for pigmentation in fishes. Indian Journal Natural Products and Resources 6(1), 46-49, 2007.

[53] Yanar, M., Zeynep, E., Hunt, A. y Hakan, B. The use of alfalfa, Medicago sativa as a natural carotenoid source in diets of goldfish, Carassius auratus. Aquaculture, 284(1-4), 196-200, 2008.

[54] Yuangsoi, B., Jintasataporn, O., Areechon, N. y Tabthipwon, $\mathrm{P}$. The pigmenting effect of different carotenoids on fancy carp (Cyprinus carpio). Aquaculture Nutrition, 17, e306-e316, 2011.

[55] Sun, X., Chang, Y., Ye, Y., Ma, Za., Liang, Y., Li, T., Jiang, N., Xing, W. y Luo, L. The effect of dietary pigments on the coloration of Japanese ornamental carp (koi, Cyprinus carpio L.). Aquaculture, 342-343, 62-68, 2012.

[56] Mandal, B., Mukherjee, A. y Banerjee, S. Growth and pigmentation development efficiencies in fantail guppy, Poecilia reticulata fed with commercially available feeds. Agriculture and Biology Journal of North America, 1(6), 1264-1267, 2010.

[57] Ituassú, D.R, Pereira, M., Roubach, R., Crescêncio, R., Cavero, B. y Gandra, A.L. Níveis de proteína bruta para juvenis de pirarucu. Pesquisa Agropecuaria Brasileira, 40(3), 255-259, 2005.

[58] Elangovan, A. y Shim, K. F. Growth response of juvenile Barbodes altus fed isocaloric diets with variable protein levels. Aquaculture, 158(3-4), 321329, 1997.

[59] Lochmann, R.T. y Phillips, H. Dietary protein requirement of juvenile golden shiners (Notemigonus crysoleucas) and goldfish (Carassius auratus) in aquaria. Aquaculture, 128(3-4), 277-285, 1994.
[60] Fiogbé, E.D. y Kestemont, P. An assessment of the protein and amino acid requirements in goldfish (Carassius auratus) larvae. Journal of Applied Ichthyology, 11(3-4), 282-289, 1995.

[61] Olvera-Novoa, M.A., Gasca-Leyva, E., y Martinez-Palacios, C.A. The dietary protein requirements of Cichlasoma synspilum Hubbs, 1935 (Pisces: Cichlidae) fry. Aquaculture Research, 27(3), 167-166, 1996.

[62] Sampaio AMBM, Kubitza F, Cyrino JEP. Relação energia: proteína na nutrição do tucunaré. Scientia Agricola, 57(2), 213-219, 2000.

[63] Zuanon, J.A., Salaro, A.L., Silveira-Moraes S.S., de Oliveira-Alves L.M., Balbino E.M. y E. Araújo, E. Dietary protein and energy requirements of juvenile freshwater angelfish. Revista Brasileira de Zootecnia, 38(6), 989-993, 2009.

[64] Chong, A.S., Hashim, R. y Ali, A.B. Dietary protein requirements for discus (Symphysodon spp.). Aquaculture Nutrition, 6(4), 275-278, 2000.

[65] Mohanta, K.N., Subramanian, S. y Korikanthimath, V.S. Replacement of fish meal protein by surimi by-product protein in the diet of blue gourami Trichogaster trichopterus fingerlings. Journal of Animal Physiology and Animal Nutrition, 97(1), 10-19, 2011.

[66] Pai, I.K, Maryem, S.A y Mohanta, KN. Development of cost effective nutritionally balanced food for freshwater ornamental fish black molly (Poecilia latipinna). Journal of Aquaculture Research \& Development, 7(02), 2-4, 2016. 